\title{
IDEMPOTENT MULTIPLIERS ON SPACES OF CONTINUOUS FUNCTIONS WITH p-SUMMABLE FOURIER TRANSFORMS
}

\author{
LYNETTE M. BLOOM AND WALTER R. BLOOM
}

\begin{abstract}
ABSTRACr. Let $G$ denote a compact abelian group, and $A^{P}$ the space of functions continuous on $G$ and having $p$-summable Fourier transforms. The idempotent multipliers from $A^{p}$ to $A^{q}$ are characterised for $p, q \in[1,2]$.
\end{abstract}

Throughout $G$ will denote a compact Hausdorff abelian group with character group $\Gamma$. Given $p \in[1,2]$ we shall write $A^{p}$ for the Banach space of functions continuous on $G$ with $p$-summable Fourier transforms, and normed by $\|f\|=$ $\|f\|_{\infty}+\|\hat{f}\|_{p}$. Note that we can identify $A^{2}$ with $C$ and $A^{1}$ with $A$, the spaces of functions on $G$ that are continuous and have absolutely convergent Fourier series respectively. An application of the Hahn-Banach theorem shows that the dual $\left(A^{p}\right)^{\prime}$ of $A^{p}$ can be identified with $M+F l^{p^{\prime}}$, where $M$ is the space of Radon measures on $G$ and $F l^{p^{\prime}}$ is the space of pseudomeasures on $G$ with Fourier transforms in $l^{p^{\prime}}$. Here $p^{\prime}$ is the exponent conjugate to $p$, that is, $p^{\prime}=p /(p-1)$ with the usual convention if $p=1$. The duality is expressed by

$$
h(f)=\mu * f(0)+\sigma * f(0), \quad f \in A^{p},
$$

where $h \in\left(A^{p}\right)^{\prime}, \mu \in M$ and $\sigma \in F l^{p^{\prime}}$.

We shall write $\left(A^{p}, A^{q}\right)$ for the set of pseudomeasures that are multipliers from $A^{p}$ to $A^{q}$; that is, $\sigma \in\left(A^{p}, A^{q}\right)$ if and only if for every $f \in A^{p}$ there exists $g \in A^{q}$ with $\sigma * f=g$. Using the above characterisation of the dual of $A^{p}$ the following result can be proved.

LEMMA 1. Given $p \in[1,2]$ and $q \in[p, 2]$,

$$
\left(A^{p}, A^{q}\right)=\left(A^{p}, A^{p}\right)=\left(A^{p}, C\right)=M+F l^{p^{\prime}} .
$$

The $A^{p}$ spaces and their multiplier spaces have been considered previously; see [1], [2], [5] and [6]. Here we are interested in characterising the set of multipliers $\phi \in\left(A^{p}, A^{q}\right)$ that are idempotent; that is, satisfy $\phi * \phi=\phi$. We shall write $\left(A^{p}, A^{q}\right)_{I}$ for the set of all idempotent elements of $\left(A^{p}, A^{q}\right)$. In addition, we shall denote by $J$ the set of all idempotent measures on $G$, by $\xi_{\Xi}$ the characteristic function of the set $\Xi$, and by $|\Xi|$ the cardinality of $\Xi$ if $\Xi$ is finite.

By considering Fourier transforms we see that to each $\phi \in\left(A^{p}, A^{q}\right)_{I}$ there corresponds unique $\Xi \subseteq \Gamma$ for which $\hat{\phi}=\xi_{\Xi}$. In the case $p=1, q \in[1,2]$, it is easy to see (using Lemma 1) that 


$$
\left(A, A^{q}\right)_{I}=(A, A)_{I}=\left\{\phi: \hat{\phi}=\xi_{\Xi} \text { for some } \Xi \subseteq \Gamma\right\} .
$$

For other values of $p, q, p<q$, the idempotent multipliers are precisely the idempotent measures.

TheOREM 1. For $p \in(1,2], q \in[p, 2],\left(A^{p}, A^{q}\right)_{I}=\left(A^{p}, A^{p}\right)_{I}=J$.

Proof. The first equality follows directly from Lemma 1 . As for the second, consider $\phi \in\left(A^{p}, A^{p}\right)_{I}$. By Lemma 1 we can write $\phi=\mu-\sigma$, where $\mu \in M$ and $\hat{\sigma} \in l^{p^{\prime}}$. Choose a positive integer $n$ satisfying $2 n<p^{\prime}<2(n+1)$. Then $\hat{\sigma}^{n+1} \in l^{2}$ and hence $\sigma^{* r} \in L^{2}$ for all $r>n+1$, where $\sigma^{* r}$ denotes the $r$-fold convolution of $\sigma$ with itself. Now

$$
\mu^{* s}=(\phi+\sigma)^{* s}=\sum_{r=0}^{s}\left(\begin{array}{l}
s \\
r
\end{array}\right) \phi^{* s-r} * \sigma^{* r}=\sum_{r=0}^{s-1}\left(\begin{array}{l}
s \\
r
\end{array}\right) \phi * \sigma^{* r}+\sigma^{* s}
$$

(recall that $\phi^{* k}=\phi$ for all $k>1$ ) and consideration of this expression for $s=n+$ $1, n+2, \ldots, 2 n+1$ gives

$$
\left[\begin{array}{cccc}
1 & \left(\begin{array}{c}
n+1 \\
1
\end{array}\right) & \cdots & \left(\begin{array}{c}
n+1 \\
n
\end{array}\right) \\
1 & \left(\begin{array}{c}
n+2 \\
1
\end{array}\right) & \ldots & \left(\begin{array}{c}
n+2 \\
n
\end{array}\right) \\
\vdots & \vdots & & \vdots \\
1 & \left(\begin{array}{c}
2 n+1 \\
1
\end{array}\right) & \cdots & \left(\begin{array}{c}
2 n+1 \\
n
\end{array}\right)
\end{array}\right]\left[\begin{array}{c}
\phi \\
\phi * \sigma \\
\vdots \\
\phi * \sigma^{* n}
\end{array}\right]=\left[\begin{array}{c}
\mu_{1} \\
\mu_{2} \\
\vdots \\
\mu_{n+1}
\end{array}\right]
$$

where $\mu_{i} \in M, i=1,2, \ldots, n+1$. The coefficient matrix is nonsingular; indeed using the identity $\left(\left(_{s}^{t+1}\right)-\left({ }_{s}^{t}\right)=\left({ }_{s-1}^{t}\right), t>s\right.$, it can be reduced to the upper triangular matrix $\left(\alpha_{i j}\right)$, where $\alpha_{i j}=\left(\begin{array}{c}n+1 \\ j-i\end{array}\right)$ for $i<j$. It follows that $\phi \in M$ and hence $\phi \in J$, thus showing that $\left(A^{p}, A^{p}\right)_{I} \subseteq J$. The reverse inclusion is clear, completing the proof of the theorem.

For $p \in(1,2], q \in[1, p)$ the only idempotent multipliers $\phi$ from $A^{p}$ to $A^{q}$ are those given by $\hat{\phi}=\xi_{\Xi}$, where $\Xi$ is finite. To prove this we require some preliminary results.

Let $\mathcal{K}$ denote the family of all cosets in $\Gamma$ and $\Re$ the Boolean ring generated by $\mathscr{K}$. Every $\Xi \in \Re$ can be written as a finite union of sets of the form $\Pi=\bigcap_{i=1}^{n} \Lambda_{i}^{c}$ $\cap \cap_{i=n+1}^{r} \Lambda_{i}$, where $\Lambda_{i} \in \mathcal{K}$ and $\Lambda_{i}^{c}$ denotes the complement of $\Lambda_{i}$. If $\Xi$ is infinite then so must be at least one such set $\Pi$.

LEMMA 2. Given an infinite set $\Pi$ of the above type there exists a finite set $\Upsilon$ with the property that $\Pi \cup \Upsilon$ contains an infinite coset.

ProOF. First we show that a finite intersection of cosets is either empty or itself a coset. Indeed consider $\Lambda_{1}, \Lambda_{2} \in \mathcal{K}$ such that $\Lambda_{1} \cap \Lambda_{2}$ is nonempty. Then $\Lambda_{1}=\eta_{1}$ $+\Omega_{1}, \Lambda_{2}=\eta_{2}+\Omega_{2}$, where $\Omega_{1}, \Omega_{2}$ are subgroups of $\Gamma$, and there exist $\chi_{1} \in \Omega_{1}$, $\chi_{2} \in \Omega_{2}$ such that $\eta_{1}+\chi_{1}=\eta_{2}+\chi_{2}$. Hence

$$
\Lambda_{1} \cap \Lambda_{2}=\left(\eta_{2}+\chi_{2}+\Omega_{1}\right) \cap\left(\eta_{2}+\chi_{2}+\Omega_{2}\right)=\eta_{2}+\chi_{2}+\Omega_{1} \cap \Omega_{2} \in \mathcal{K} .
$$


Thus a nonempty intersection of two cosets is itself a coset and the result extends to arbitrary finite intersections by induction.

Applying this to (the nonempty set) $\Pi$ we see that

$$
\Pi=\bigcap_{i=1}^{n} \Lambda_{i}^{c} \cap \Lambda, \quad \Lambda_{i}, \Lambda \in \mathscr{K} .
$$

By relabelling if necessary we can assume that $\Pi \cup \Upsilon$ contains the infinite set $\bigcap_{i=1}^{n} \Lambda_{i}^{c} \cap \Lambda$, where $\Upsilon$ is finite and each $\Lambda_{i}$ is infinite. Now $\Lambda_{i}=\eta_{i}+\Omega_{i}$, $\Lambda=\eta+\Omega$ for some subgroups $\Omega_{i}, \Omega$ of $\Gamma$. We consider the following cases:

(1) $\cap_{i=1}^{n} \Omega_{i} \cap \Omega$ is infinite. Then $\Pi \cup \Upsilon$ contains $\cap_{i=1}^{n} \Lambda_{i}^{\prime} \cap \Lambda$ which, for suitable cosets $\Lambda_{i}^{\prime}$ of $\Omega_{i}$, is nonempty; just use the fact that each $\Lambda_{i}^{c}$ is a union of cosets of $\Omega_{i}$. By the argument used in the first part of the proof of the lemma, $\cap_{i=1}^{n} \Lambda_{i}^{\prime} \cap \Lambda$ is an infinite coset in $\Gamma$.

(2) $\cap_{i=1}^{n} \Omega_{i} \cap \Omega$ is finite, $\cap_{i \in I} \Omega_{i} \cap \Omega$ is infinite for some $I \subseteq\{1,2, \ldots, n\}$ with $|I|=n-1$. Then there are cosets $\Lambda_{i}^{\prime \prime}$ of $\Omega_{i}$ such that $\Pi \cup \Upsilon$ contains the nonempty set $\cap_{i \in I} \Lambda_{i}^{\prime \prime} \cap \Lambda \cap \Lambda_{j}^{c}$, where $\{j\}=\{1,2, \ldots, n\} \backslash I$, and $\Upsilon^{\prime}=$ $\cap_{i \in I} \Lambda_{i}^{\prime \prime} \cap \Lambda \cap \Lambda_{j}$ is (empty or) finite. Thus $\Pi \cup \Upsilon \cup \Upsilon^{\prime} \supseteq \cap_{i \in I} \Lambda_{i}^{\prime \prime} \cap \Lambda$, an infinite coset.

(3) $\cap_{i \in I} \Omega_{i} \cap \Omega$ is finite for all $I \subseteq\{1,2, \ldots, n\}$ with $|I|=n-1$. In this case we return to (2) above and repeat the process.

After $n-1$ steps we have that $\Omega_{i} \cap \Omega$ is finite for each $i \in\{1,2, \ldots, n\}$ and

$$
\Pi \cup \Upsilon \cup \bigcup_{i=1}^{n}\left(\Lambda_{i} \cap \Lambda\right) \supseteq\left(\bigcap_{i=1}^{n} \Lambda_{i}^{c} \cap \Lambda\right) \cup\left(\bigcup_{i=1}^{n} \Lambda_{i} \cap \Lambda\right)=\Lambda
$$

is the required infinite coset.

TheORem 2. Let $\Xi \in \Re$ be infinite and $p \in(1,2]$. Then there exists a continuous function $f$ with supp $\hat{f} \subseteq \Xi$ and $\hat{f} \in l^{p} \backslash l^{q}$ for all $q \in[1, p)$.

Proof. By Lemma 2 we have the existence of a finite set $\Upsilon$ such that $\Xi \cup \Upsilon$ contains an infinite coset $\Lambda$. We shall construct $f$ as in the statement of the theorem with supp $\hat{f} \subseteq \Lambda$, and then the result will follow. There are two cases:

(a) The subgroup $\Omega=-\eta+\Lambda$ has an element $\chi$ of infinite order. Now construct Rudin-Shapiro polynomials $f_{n}$ in the usual way ([7, (37.19)]) so that $\hat{f}_{n}$ takes values in $\{-1,0,1\}$,

$$
\operatorname{supp} \hat{f}_{n}=\left\{0, \chi, \ldots,\left(2^{n}-1\right) \chi\right\} \text { and }\left\|f_{n}\right\|_{\infty}<2^{(n+1) / 2} \text {. }
$$

Choose $\chi_{n} \in \Omega$ so that the spectra of the functions $\chi_{n} f_{n}$ are pairwise disjoint and put $f=\sum_{n=0}^{\infty}(n+1)^{-2} 2^{-n / p_{\eta}} \chi_{n} f_{n}$. Then

$$
\sum_{n=0}^{\infty}(n+1)^{-2} 2^{-n / p}\left\|\eta \chi_{n} f_{n}\right\|_{\infty}<\infty,
$$

so that $f \in C$. Clearly supp $\hat{f} \subseteq \Lambda$. That $f \in A^{p}$ follows from the estimate

$$
\|\hat{f}\|_{p}<\sum_{n=0}^{\infty}(n+1)^{-2} 2^{-n / p} 2^{n / p}<\infty,
$$

whereas $\|\hat{f}\|_{q}^{q}=\sum_{n=0}^{\infty}(n+1)^{-2 q} 2^{-n q / p} 2^{n}$ shows that $f \notin A^{q}$ for any $q<p$. 
(b) $-\eta+\Lambda=\Omega$ is a torsion subgroup of $\Gamma$, in which case there is a strictly increasing sequence $\left(\Omega_{n}\right)$ of finite subgroups of $\Omega$. For each $n$ construct $f_{n}$ (as in [4]) with the properties $\left|\hat{f}_{n}\right|=1$ on $\Omega_{n}$, supp $\hat{f}_{n}=\Omega_{n}$ and $\left\|f_{n}\right\|_{\infty}=\left|\Omega_{n}\right|^{1 / 2}$. As in (a) choose $\chi_{n} \in \Omega$ so that the spectra of the functions $\chi_{n} f_{n}$ are pairwise disjoint and put $f=\sum_{n=0}^{\infty}(n+1)^{-2} 2^{-n / p_{\eta}} \chi_{n} f_{n}$.

Theorem 3. Let $p \in(1,2], q \in[1, p)$ be given. Then $\phi \in\left(A^{p}, A^{q}\right)_{I}$ if and only if $\hat{\phi}=\xi_{\Xi}$ for some finite set $\Xi \subseteq \Gamma$.

Proof. Sufficiency is obvious. As for the other direction, consider $\phi \in$ $\left(A^{p}, A^{q}\right)_{I}$. Using $\left(A^{p}, A^{q}\right)_{I} \subseteq\left(A^{p}, A^{p}\right)_{I}$ we see from Theorem 1 that $\phi \in J$ and hence, by [3, Theorem 3], $\hat{\phi}=\xi_{\Xi}$ for some set $\Xi$ belonging to the coset ring $\Re$ of $\Gamma$. Suppose now that $\Xi$ is infinite. By Theorem 2 there exists $f \in C$ with supp $\hat{f} \subseteq \Xi$ and $\hat{f} \in l^{p} \backslash l^{q}$. This contradicts the assumption that $\phi \in\left(A^{p}, A^{q}\right)_{I}$.

Corollary. Suppose $G$ is infinite and let $p \in(1,2], q \in[1, p)$ be given. Then $\left(A^{p}, A^{q}\right)_{I} \subsetneq\left(A^{p}, A^{p}\right)_{I}$.

Proof. That this inclusion is strict follows from Theorem 3 together with the fact that $\delta_{0}$ (the Dirac measure at 0 ) belongs to $\left(A^{p}, A^{p}\right)_{I}$.

Acknowledgement. We should like to thank Dr. John F. Price for suggesting some of the problems considered in this paper.

\section{REFERENCES}

1. Lynette M. Bloom, The Fourier multiplier problem for spaces of continuous functions with p-summable transforms, J. Austral. Math. Soc. 17 (1974), 319-331.

2. Lynette M. Butler, Certain nonalgebras in harmonic analysis, Bull. Austral. Math. Soc. 4 (1971), 247-254.

3. Paul J. Cohen, On a conjecture of Littlewood and idempotent measures, Amer. J. Math. 82 (1960), 191-212.

4. Colin C. Graham, The Sidon constant of a finite abelian group, Proc. Amer. Math. Soc. 68 (1978), 83-84.

5. Michael B. Gregory, p-Helson sets, $1<p<2$, Israel J. Math. 12 (1972), 356-368.

6. Edwin Hewitt and Gunter Ritter, Uber die Integrierbarkeit von Fourier-Transformierten auf Gruppen. Teil 1. Stetige Funktionen mit kompaktem Träger und eine Bemerkung über hyperbolische Differentialoperatoren, Math. Ann. 224 (1976), 77-96.

7. Edwin Hewitt and Kenneth A. Ross, Abstract harmonic analysis. Vol. II, Die Grundlehren der Math. Wissenschaften, Band 152, Springer-Verlag, Berlin and New York, 1970.

School of Mathematical and Physical Sciences, Murdoch University, Murdoch, Westeren Australi, 6150, Australia 